\title{
Panorama actual en la gestión del agua y saneamiento en Argentina
}

\author{
Verónica L. Cáceres \\ Investigadora-Docente del Instituto del Desarrollo Humano, Área de Economía, \\ Universidad Nacional de General Sarmiento, Argentina. \\ vcaceres@ungs.edu.ar
}

Fecha de recepción: 07/12/2015 Fecha de aceptación: 23/05/2016

\section{Resumen}

Se muestra un panorama de la gestión de los servicios de agua y saneamiento en Argentina entrado el siglo XXI. Estos servicios experimentaron una transformación profunda en el país de la mano de los procesos de descentralización, privatización y reestatización. Dichos procesos fueron cuestionados por el debate sobre el reconocimiento del acceso al agua como un derecho humano inalienable y no como una simple mercancía. La gestión de servicios de agua y saneamiento involucra la gestión del recurso hídrico estratégico, crecientemente escaso, demandado por otros usos y a un conjunto de infraestructuras críticas. El artículo primero problematiza estas características, luego da cuenta de la evolución histórica de los modelos de gestión de los servicios en el país, su gestión actual y los desafíos centrales que asume.

\section{Palabras claves}

Sudamérica, recursos hídricos, infraestructura, gestión del agua

\begin{abstract}
The paper provides an overview of the management of water and sanitation services in Argentina in the beginning of the XXI century. These services have experienced an intensive transformation from the process of decentralization, privatization and renationalization in the country. These processes were over passed by the debate about the recognition of water access as an inalienable human right and not as a simple commodity. The management of these services involves the strategic management of water resource, increasingly scarce, demanded for other uses and a set of critical infrastructure. Firstly, the paper challenges these features; then it gives an account of the development of the service management models in the country; Later, the current management of services and the central challenges that it assumes.
\end{abstract}

\section{Keywords}

South America, water resource, infrastructure, water management 


\section{Introducción}

Argentina es un país que asume la forma federal de gobierno, tiene 23 provincias más la Ciudad Autónoma de Buenos Aires (CABA) y para 2010, su población superó los 40 millones.

Los servicios de agua y saneamiento se desarrollaron en el país como parte de las políticas que apuntaron a mejorar la salubridad pública, desde fines del siglo XIX, y en las últimas dos décadas del siglo $\mathrm{XX}$ experimentaron una transformación profunda, primero de la mano de la descentralización y segundo, por la masiva introducción de capitales privados en la gestión. La descentralización y privatización se observó también en distintas experiencias en los países de América Latina y el Caribe.

Hasta 1980 los servicios se encontraban a cargo de Obras Sanitarias de la Nación (OSN), empresa insignia del sector, en la mayor parte del país desde su creación en 1912. Luego la responsabilidad sobre la política sectorial recae sobre los Estados provinciales y por decisión de estos últimos, en algunos casos también sobre ciertos municipios. Esto es así en todo el territorio nacional a excepción de una parte conformada por 17 municipios del Conurbano Bonaerense y la CABA que se encuentran aún bajo jurisdicción del Estado nacional.

Asimismo el esquema de organización federal adoptado en el país reconoce la preexistencia de las provincias a la nación, por ello los Estados provinciales conservan las competencias no delegadas por la Constitución al gobierno federal. Con la reforma constitucional de 1994 quedó establecido que, en materia ambiental, corresponde al Estado nacional la responsabilidad de "dictar las normas que contengan los presupuestos mínimos de protección" y a los Estados provinciales que tienen "el dominio originario de los recursos naturales existentes en su territorio", comple- mentarias (Constitución Nacional, artículos 41 y 124$){ }^{1}$

Al estar los servicios de agua y saneamiento descentralizados y constitucionalmente las provincias contar con el dominio originario de sus recursos naturales no existe una Ley $\mathrm{Na}$ cional del Agua, como sucede en México, que fije lineamientos de la política hídrica en todo el territorio.

Por el contrario existe un conjunto de normativas dispersas que regulan de forma directa o indirecta distintas aristas de los recursos hídricos, como la Ley de Régimen de Gestión Ambiental de Aguas $N^{\circ} 25.688$, sancionada en 2002, que legisla en materia de cuencas interjuridisdiccionales y crea los comités de cuencas como organismos federales de asesoramiento.

La Ley de Régimen de Gestión Ambiental de Aguas $N^{\circ} 25.688$ aún no ha sido reglamentada por el Poder Ejecutivo Nacional. Asimismo es el Consejo Hídrico Federal la única instancia federal de concertación y coordinación de la política hídrica federal (con la sanción de la Ley $N^{\circ}$ 26.438). Dicho Consejo está integrado por el Estado Nacional y todas las jurisdicciones que se encuentran divididas en los siguiente Consejos Regionales COHINOA (Catamarca, Jujuy, Salta, Santiago del Estero y Tucumán), COHINEA (Chaco, Formosa y Misiones), COHICU (La Rioja, Mendoza, San Juan y San Luis"; COHILI (Santa Fe, Entre Ríos y Corrientes), COHICEN (Buenos Aires (PBA), Córdoba, La Pampa y la CABA), COHIPA (Chubut, Neuquén, Río Negro, Santa Cruz y Tierra del Fuego e Islas del Atlántico Sur).

A los cambios que experimentaron los servicios de agua y saneamiento con la descentralización y posterior privatización se agregan otros con el proceso de re-estatizaciones, en

1 El Estado nacional ejerce jurisdicción sobre la navegación, el comercio interprovincial e internacional, las relaciones internacionales, la celebración de tratados internacionales. 
el marco de una fuerte crisis económica y social que experimentó el país a inicios del presente siglo.

Este trabajo pretende contribuir, puntualmente, a la reflexión sobre ¿cómo es el escenario de los servicios de agua y saneamiento tras las re-estatizaciones en Argentina? ¿Cuáles son los principales desafíos que presenta la gestión de los servicios?

En primer lugar se destacan las características que tiene la gestión de los servicios de agua y saneamiento y su relación con la gestión hídrica en general; luego se da cuenta de la evolución histórica de los servicios en Argentina y en la región; para luego centrarnos en las particularidades actuales que tiene la provisión y regulación de los servicios y, ya en las conclusiones, dar cuenta de los principales desafíos.

\section{La gestión de un recurso natural estratégico}

La gestión de los servicios públicos de agua y saneamiento involucra un conjunto complejo de infraestructuras que forman parte de los servicios urbanos provistos mediante redes físicas con prestaciones colectivas que ofrecen medios para la producción y reproducción de la vida y la satisfacción de necesidades (Mutuberría, 2007). En términos económicos se trata de dos monopolios naturales diferenciables por las redes de tuberías y drenaje y de las grandes obras de potabilización del agua y depuración de los residuos, así como de los acueductos y drenajes que conforman la infraestructura típica de las zonas urbanas.

La prestación de estos servicios involucra la gestión de un recurso natural estratégico que no tiene sustitutos, que es vulnerable y crecientemente escaso y cumple funciones ambientales, sociales, económicas y culturales. Aunque el $70 \%$ del planeta está cubierto por agua sólo el $2,5 \%$ es dulce y gran parte de esta última se encuentra congelada en glaciares y el resto es salada (Comisión Nacional del Agua, 2003). En las últimas cinco décadas el consumo de agua se triplicó y para 2025 se estima que el 35\% de la población se verá afectada por estrés hídrico (Buch, 2013).

El agua cumple funciones indispensables para la vida, entre las que se destacan que al actuar como solvente permite el transporte de sustancias nutritivas hasta las células así como de sustancias de desecho desde las células hacia el exterior, etc. (Bella y Hure, 2007). A diferencia de otros recursos naturales, el agua dulce no tiene sustituto: si se agota o se contamina y no se le efectúan los tratamientos para recuperarla, se pierde.

Aunque existen técnicas para desalinizar el agua de océanos o mares estas son complejas, costosas y, entre otras cosas, "aún no se ha encontrado el modo de deshacerse de la salmuera que queda del proceso y de los elementos químicos que se utilizan en el mismo y para el funcionamiento correcto de la planta, sin afectar al medio ambiente" (Bruzone, 2009:17). ${ }^{2}$

Entre las particularidades del agua, Solanes y Jouravlev (2005) destacan: la movilidad e incertidumbre y los problemas de establecer derechos de propiedad; la existencia de economías de escala (en el caso de los servicios por redes en las fases de almacenamiento, transporte, distribución y recolección y tratamiento de efluentes); la diversidad de usos; la interdependencia general de los usuarios y la naturaleza unidireccional y asimétrica de las interrelaciones e interdependencia entre los usos y usuarios de agua en un sistema hídrico integrado (que dificulta el uso equitativo y eficiente en términos sociales, económicos y ambientales).

El agua dulce se encuentra desigualmente distribuida en el planeta y los reservorios más importantes están en América del Sur. Esta región se convierte así en un lugar estratégico por la disponibilidad del recurso, ya sea por la exis-

2 Entre los casos de desalinización del agua más importantes se encuentran distintos proyectos en Israel. 
tencia de Cuencas como la de Orinoco, Esteros como los de Ibera y Acuíferos como el Guaraní.

En Argentina dos tercios de su territorio están conformados por regiones áridas y semiáridas y un tercio tiene abundantes fuentes de agua, fundamentalmente superficiales que representan al $84 \%$ de las disponibilidades hídricas del país.

Aunque la oferta hídrica media anual del país es de los $20.000 \mathrm{~m}^{3} /$ habitantes, por encima del nivel de estrés hídrico adoptado por el programa de la Naciones Unidas para el desarrollo (equivalente a una disponibilidad de $1.000 \mathrm{~m}^{3 /}$ habitantes) hay un desbalance entre las demandas y disponibilidad, en tanto la oferta se concentra fuertemente en la región que integra la cuenca del Río de la Plata (Rodríguez, 2008).

Entre los principales problemas hídrico-ambientales que enfrentan las distintas regiones de Argentina se encuentran, en primer lugar, aquellos derivados de la falta de tratamiento de efluentes (industriales, domiciliarios y urbanos en general) y la insuficiente planificación urbana. En segundo, la contaminación del agua subterránea, cuya gestión corresponde a las jurisdicciones provinciales, y el tercero, la degradación de humedales por la contaminación, construcción de sistemas de desagüe, canalizaciones clandestinas que modifican el escurrimiento superficial, etc. (Academias Nacionales de Ingeniería, Ciencias Económicas y Ciencias Exactas, Físicas y Naturales, 2011).

Es de destacar que la gestión de los servicios de agua y saneamiento involucra un recurso natural demandado por otros usos (que tienden a competir entre sí, especialmente en zonas áridas o semiáridas) que se despliegan en dispositivos normativos y de gestión particulares, donde la provisión doméstica constituye una forma de aprovechamiento en la que intervienen un entramado de actores estatales y no estatales que actúan en un contexto determinado donde movilizan sus recursos e intereses.
Por lo cual, resulta relevante que la política del agua y saneamiento se vincule de forma integral con la política hídrica considerando a las cuencas como unidad, es decir a la zona natural de la superficie terrestre donde el agua de lluvia tiende a ser drenada a través de una red de corrientes que confluyen a una corriente principal y por ésta hacia un punto en la salida de dicha superficie. Normalmente dicha corriente principal da nombre a la cuenca correspondiente. Las grandes cuencas vertientes se forman por estas cuencas" (Abihaggle y Day, 2004: 22).

Mientras el objeto de la política de agua y saneamiento es el abastecimiento doméstico de la población, el de la hídrica incluye además al agua como recurso natural; bien social cuya existencia es condición básica para el asentamiento de las poblaciones; bien económico ya que es un insumo para la producción y el turismo y como "mal sobrante" en las inundaciones (Abihaggle y Day, 2004). Es decir considera los usos doméstico, agrícola, industrial, mineral, petrolero, hidroeléctrico, turístico y ecológico, etc.

\section{Los modelos de gestión de los servicios en Argentina y en la región}

En Argentina los servicios de agua y saneamiento se conformaron en las últimas décadas del siglo XIX como parte de las políticas sanitarias y de control de las epidemias de cólera y fiebre amarilla y se expandieron, fundamentalmente, a partir de la intervención del Estado nacional. Los servicios estuvieron centralizados, mayormente en la órbita de OSN, empresa estatal insignia del sector creada en 1912 y que pasó a prestar los servicios en las principales ciudades del país.

Esta situación se dio en otros países de la región donde los servicios estuvieron a cargo de organismos estatales no sólo en la prestación sino en la construcción de las grandes obras de infraestructura (plantas de potabilización, duc- 
tos de distribución, acueductos, etc.), como en Paraguay, Colombia y Bolivia, entre otros.

Ya para la década del setenta del siglo XX, en un marco de inclusión de los problemas ambientales en la agenda internacional, comenzó a discutirse también la problemática del acceso al agua y la protección de los recursos hídricos.

Es de destacar que en plena retracción de las políticas económicas keynesianas de fuerte intervención estatal en la economía, a inicios de la década del ochenta, cobraron relevancia los discursos pro-descentralización de los servicios públicos, especialmente desde los organismos internacionales de crédito como el FMI (así como sucedió en otras arenas políticas).

La descentralización y transferencia de los servicios de agua y saneamiento a los niveles subnacionales de gobierno (provincias) y, por decisión de estos, a algunos municipios, incluyó como promesa la mejora de la eficiencia y la posibilidad de "acercar la solución de los problemas locales a los niveles más cercanos a la población" en el marco del principio de subsidiariedad del Estado (Rojas, 2010).

Así esto derivó en la presencia de modelos de gestión pública de menor alcance a cargo de los gobiernos subnacionales (provinciales, regionales o estaduales y/ o locales), donde se destacaron, por un lado, la transferencia y provisión directa por parte de los municipios en Colombia, Perú, Bolivia y Ecuador. Por otro, la implementación de esquemas mixtos en Argentina y Chile, en los que de un modelo de provisión centralizado en la mayoría del territorio se pasó a la coexistencia de distintos modelos por la transferencia de competencias a los niveles provinciales/regionales y por decisión de estos últimos en algunos casos a los municipios.

En otros países, como en México y Brasil, la creación de empresas estaduales surgió luego que los municipios (a los que primero se les confirió la provisión) enfrentaron problemas para responder a estas tareas.

Más allá de las promesas, la lógica de la descentralización en el sector obedeció mayormente a la búsqueda de equilibrio fiscal en las arcas públicas nacionales y la misma no tendió a considerar las características particulares del sector (como las economías de escala, las necesidades de financiamiento, etc.). Esta situación adquirió notoriedad en Argentina con la transferencia a los niveles subnacionales que, con excepción de la PBA, contaban con escasa experiencia en la gestión de servicios de infraestructura. La PBA es la provincia más importante del país en términos poblacionales y económicos.

El proceso de descentralización coincidió con el "Decenio internacional del agua potable y el saneamiento ambiental (1981-1991)" proclamado por la Asamblea General de las Naciones Unidas a fines de 1980.

Ya para la década del noventa, en pleno auge del paradigma neoliberal que ciertamente afectó a distintas arenas de políticas públicas, la problemática del acceso al agua y saneamiento formó parte de numerosas disposiciones en declaraciones y programas aprobados por la ONU.

A partir de la Agenda 21 y de la Cumbre Mundial sobre Desarrollo Sostenible, llevada adelante en Río en 1992, los organismos internacionales comenzaron hablar de la importancia de la gestión del agua de forma integral y no sólo sectorial.

El concepto de Gestión Integrada de los Recursos Hídricos presenta distintas definiciones aunque una de las más difundidas entiende que se trata de "un proceso que promueve la gestión y el desarrollo coordinado del agua, el suelo y los otros recursos relacionados, con el fin de maximizar los resultados económicos y el bienestar social de forma equitativa sin 
comprometer la sostenibilidad de los ecosistemas vitales" (Global Water Partnership, 2008).

Desde esta óptica se promovió una mirada de la gestión del agua basada en tres ideas básicas: la integración sectorial, la descentralización territorial y la participación de la sociedad civil (Abers, 2010).

Asimismo en 1992 tras la Declaración de Dublín sobre el Agua y el Desarrollo sostenible que se dio como conclusión de la Conferencia Internacional sobre el Agua y el Medio Ambiente, realizada en la Ciudad de Dublín, Irlanda; de la cual participaron los gobiernos de 100 países, incluyendo a la Argentina, y representantes de 80 organizaciones internacionales, se acordaron los siguientes principios rectores: "1-. El agua dulce es un recurso finito y vulnerable, esencial para sostener la vida, el desarrollo y el medio ambiente. 2-. El aprovechamiento y la gestión del agua debe inspirarse en un planteamiento basado en la participación de los usuarios, los planificadores y los responsables de las decisiones a todos los niveles. 3-. La mujer desempeña un papel fundamental en el abastecimiento, la gestión y la protección del agua. 4-. El agua tiene un valor económico en todos sus diversos usos en competencia a los que se destina y debería reconocérsele como un bien económico" ${ }^{\prime 3}$.

A la par de estos cambios en las concepciones generales de la gestión del agua comenzó a promoverse también la inclusión de capitales privados en la provisión de los servicios bajo la pretensión que esto favorecería la universalización, la protección de los recursos hídricos, el aumento de la eficiencia y la mejora general de la calidad. En tanto se esperaba que "la participación de operadores privados internacionales aportaran financiamiento y conocimientos de gestión gerencial y tecnológica para resolver las deficiencias que afectaban la prestación de estos servicios" (Lentini \& Ferro, 2014:12).

3 Para mayor detalle ver http://www.wmo.int/pages/prog/ hwrp/documents/espanol/icwedecs.html
El traspaso de los servicios de agua y saneamiento a empresas privadas acaecidas desde los primeros años de la década del noventa del siglo pasado en la región en general y en Argentina en particular dieron lugar a modelos de gestión de los servicios que se sustentaron en la concepción del agua como una mercancía, con prioridad en el valor económico -derivado de su carácter escaso- y su asignación mediante las leyes del mercado. ${ }^{4}$

Esta perspectiva fue promovida por distintos organismos internacionales de crédito y agentes involucrados en la industria del agua y alcanzó a objetivarse en la privatización de las prestatarias de los servicios en gran parte de la región latinoamericana (así como en otros países europeos y africanos).

Luego de una década en que la mercantilización del agua no arrojó los resultados positivos esperados en materia de los objetivos sociales prometidos (universalización de los servicios, mejora de la calidad, etc.) y por el contrario expuso a la población a situaciones de riesgo sanitario, especialmente en los países más pobres del mundo; tuvo lugar un proceso de re-estatizaciones y de retorno a modelos de gestión públicos que heredaron la institucionalidad previa.

A la par de estos eventos, el acceso a los servicios fue incluido dentro de los Objetivos de Desarrollo del Milenio, establecidos por el Comité de Derechos Económicos, Sociales y Culturales (CDESC), los cuales contemplaban la meta de reducir a la mitad la cantidad de personas existentes que carecen de acceso al agua potable $y$, también, de modo comple-

$4 \quad$ El proceso de privatizaciones en Argentina se inició en la década del noventa y alcanzó las diversas jurisdicciones: la provincia de Corrientes en 1991 se convirtió en pionera en llevar adelante la privatización de los servicios prestados hasta entonces por la Administración de Obras Sanitarias de la Provincia de Corrientes. De igual manera, el Estado nacional concesionó, de forma integral la histórica OSN. La experiencia se repitió también en Formosa (1995); Tucumán (1995); Santa Fe (1995); Santiago del Estero (1997); Córdoba (1997, sólo el servicio de agua); Mendoza (1998); Salta (1998); Buenos Aires (1999, 2000); Misiones (1999); Catamarca (2000) y La Rioja (2002). 
mentario al saneamiento (Objetivo de Desarrollo del Milenio 7).

En 2002 el CDESC emitió la Observación General $N^{\circ} 15$ que reconoció el acceso al agua como un derecho humano inalienable. Más allá de lo cual, en los siguientes años, se avanzó de forma parcial en el reconocimiento del derecho al agua y los actores internacionales que dominaban la industria del agua continuaron promoviendo su mercantilización como vía de acceso.

Ya en julio de 2010, durante la Sesión N 64 de la ONU, 122 países entre ellos Argentina, de los 192 que participan, con 29 ausentes y 41 abstenciones votaron afirmativamente por el reconocimiento del derecho al agua potable y al saneamiento como derechos humanos esenciales.

Puntualmente se declaró al "derecho al agua potable y al saneamiento como un derecho humano esencial para el pleno disfrute de la vida y de todos los derechos humanos" y se exhortó "...a los Estados y las organizaciones internacionales a que proporcionen recursos y propicien el aumento de la capacidad y la transferencia de tecnología por medio de la asistencia y la cooperación internacionales, en particular a los países en desarrollo, a fin de intensificar los esfuerzos por proporcionar a toda la población un acceso económico al agua potable y el saneamiento" (Artículos 1 y 2, Resolución N64/292).

En síntesis la historia institucional del sector de agua y saneamiento en Argentina ha seguido de cerca ciertas tendencias internacionales vinculadas a la gestión del recurso hídrico y las tensiones sobre el acceso. Los modelos de gestión que han predominado son de gestión pública centralizada o descentralizada en la que el Estado a través de organizaciones gubernamentales o empresas públicas tuvo a su cargo todas las fases de la prestación del servicio, incluyendo su regulación, control y fiscalización; y de gestión privada o mixta donde empresas privadas se hicieron cargo de la prestación y el Estado asume la regulación, control y fiscalización (Pirez (2001); Hantke-Domas \& Jouravlev (2011).

\section{Panorama actual de los servicios en Argentina}

\subsection{Las prestatarias de los servicios}

Transcurrido una década y media del siglo XXI, los servicios de agua y saneamiento en Argentina, al igual que sucede en la región, se caracterizan por la presencia de un conjunto heterogéneo de prestadores bajo distintas formas jurídicas que incluyen desde grandes empresas con características de sociedades anónimas con mayoría accionaria pública, concesiones privadas, sociedades del Estado y cooperativas, entre otras; y agencias de regulación cuya función apunta a regular, controlar y fiscalizar la prestación.

En el caso de Argentina, considerando la prestataria principal de cada jurisdicción del país, se puede señalar que el $41,6 \%$ son sociedades anónimas (SA) con mayoría accionaria del Estado, verificándose la participación de los empleados a través de un Programa de Propiedad Participada como accionistas minoritarios con un $10 \%$ de las acciones. En este caso se trata de empresas que se encuentran alcanzadas por la Ley nacional $N^{\circ} 19.550$ de sociedades comerciales y sus directivos son elegidos por períodos concretos.

Esta situación se encuentra presente en la mayor parte del Área Metropolitana de Buenos Aires (AMBA) en la que actúa Aguas y Saneamiento Argentina S.A. en 17 municipios del Conurbano Bonaerense y la Ciudad de Buenos Aires que se encuentran aún bajo jurisdicción del Estado nacional. Así también en la PBA donde actúa Aguas Bonaerense S.A.; en Santa Fe donde opera Aguas Santafesinas S.A., en Mendoza con Agua y Saneamiento Mendoza S.A., en Río Negro con Aguas Rionegrinas S.A., en Catamarca con Aguas de Catamarca, en Salta con Aguas del Norte, y en Tucumán con Sociedad Aguas del Tucumán S.A. 
Por otro lado, aún un $25 \%$ de las provincias mantienen como prestatarias principales a sociedades anónimas privadas que han accedido a la prestación de los servicios a través de Contratos de Concesión en Licitaciones Públicas. Sus accionistas mayoritarios son capitales privados, los empleados también participan a través de un Programa de Propiedad Participada con el $10 \%$ de las acciones.

Se trata de operadoras que recibieron la concesión en la década del noventa del siglo pasado y que usufructúan la tenencia de los bienes afectados a los servicios debiendo reintegrarlos a la finalización del contrato de concesión, incluyendo las mejoras que hayan realizado durante el período de concesión. Esta situación se observa en La Rioja con Aguas de la Rioja S.A., en Santiago del Estero con Aguas de Santiago S.A., en Corrientes con Aguas de Corrientes S.A.; en Formosa con Aguas de Formosa S.A., en Misiones con el Servicio de Aguas de Misiones S.A. y en Córdoba con Aguas Cordobesas S.A.

Asimismo hay un conjunto de provincias en la que las prestatarias de los servicios son cooperativas, alcanzan al 20,83\% que refieren organizaciones comunitarias basadas en los principios de no discriminación y solidaridad que se rigen por la Ley nacional $N^{\circ} 20.337$. Estas actúan en localidades de mediana y pequeña envergadura (menores de 60.000 habitantes) y en la mayoría de los casos brindan otros servicios públicos, como energía eléctrica, telecomunicaciones y gas. Las obras son soportadas económicamente por los socios a través de recursos propios o financiamiento externo. Esta situación se encuentra en las provincias de Entre Ríos, Chubut, La Pampa, Neuquén, Santa Cruz y Tierra del Fuego.

Por último se observa también la presencia de sociedades del Estado y direcciones provinciales en San Luis con la empresa San Luis Agua Sociedad del Estado y en Chaco con Servicio de agua y mantenimiento Empresa del Estado Provincial Provincia de Chaco. Se trata de estructuras empresariales cuyo único accionista es el Estado provincial y aunque tienen autarquía y autonomía económica sus directores son elegidos por el poder político.

Lo señalado en esta sección permite observar que más allá que Argentina experimentó un importante proceso de privatizaciones, actualmente la mayor parte de la población se encuentra en áreas a cargo de empresas u otros organismos públicos. Esta retirada de empresas privadas se observa también en la región en general desde los primeros años del 2000. Ducci (2007) explica que esta situación se dio por distintos motivos, entre los que destacan: decisiones estratégicas a nivel mundial del grupo controlador de las empresas ajenas a los servicios y en búsqueda de mayores ganancias (en los casos de ESVAL S.A, controlada por Anglian Water y ESSBIO S.A y Aguas Nuevo Sur Maule S.A. controlada por Thames Water en Chile y en Obras Sanitarias de Mendoza S.A. controlada por Azurix en Mendoza, Argentina); por cambios en las políticas sectoriales nacionales los casos de Aguas de Monagas S.A. controlada Fomento de Construcciones y Contratas S.A. por Estado Monagas, HIDRO LARA por Aguas de Valencia S.A. en el Estado de Lara, Venezuela; Uragua S.A. controlada por Aguas de Bilbao S.A. y Aguas de la Costa S.A. controlada por Aguas de Barcelona S.A en Uruguay; por conflictos sociales y políticos en los casos de Aguas del Tunari S.A controlada por Bechtel en Cochabamba, de Cia. Aguas del Aconquija S.A. de Compagnie Générale des Eaux en Tucumán y Aguas del Illimani S.A. de Suez); y por el quiebre del equilibrio económico-financiero del contrato en los casos de Azurix Buenos Aires S.A. controlada por Azurix, Aguas Provinciales de Santa Fe S.A; Aguas Argentinas S.A.; y Aguas Cordobesas S.A. controladas por Suez/ Aguas de Barcelona S.A). 


\subsection{La regulación de los servicios}

La literatura especializada ha centrado la atención en las funciones de regulación, control y fiscalización, especialmente desde la emergencia de actores privados en la prestación de los servicios. ${ }^{5}$ Desde fines de los años ochenta se enfatiza en la importancia que asume la regulación en post de orientar las acciones y las decisiones de los actores privados en beneficio de los intereses sociales (Corrales, 1998). El control y la fiscalización del cumplimiento de objetivos, metas y de condiciones de prestación dispuesta en las normativas vigentes.

Aunque en Argentina y la región, a excepción de Chile ${ }^{6}$, en general se observa una importante salida masiva de empresas privadas, los organismos de regulación creados en el proceso de privatizaciones permanecen en actividad y en líneas generales también sus dispositivos normativos, por lo que aún encontramos entes de regulación y marcos regulatorios diferentes en cada jurisdicción. Esto resulta llamativo en tanto fueron conformadas para regular el desempeño de empresas privadas, y que con cambios menores, pasaron a ser utilizados para regular empresas públicas.

Esta situación difiere de lo que sucede en otros países de federales como Brasil donde si bien se observa mayoría de prestadores públicos y organismos reguladores por jurisdicción, también existe una ley federal que regula los servicios y propone principios comunes en los organismos reguladores como la independencia en la toma de decisiones, "la transparencia, las capacidades técnicas, la velocidad y la objetividad de las decisiones" (artículo 21 Ley Federal 11.445 de 2007). En el caso de México aunque

$5 \quad$ El abordaje de la regulación de los servicios que adoptamos se basa en los desarrollos teóricos efectuados por Vickers y Yarrow (1991); Abdala y Spiller (1999), Solanes (1999), López y Felder (2006), entre otros.

6 En Chile, más allá de la salida de dos operadores, los servicios mayoritariamente continúan siendo prestados por empresas privadas en las distintas regiones, y la regulación se encuentra a cargo de la Superintendencia de Servicios Sanitarios. los servicios son prestados por los municipios o los estados, la propiedad de las aguas es de la nación y es administrado mediante la Comisión Nacional del Agua, pero no se ha conformado un organismo de regulación de los servicios.

Por su parte, como señalamos, en Argentina las regulaciones de los servicios dependen de las normativas de cada jurisdicción, sancionadas por leyes y/o decretos del Poder Ejecutivo (PE). Estas coexisten con un heterogéneo conjunto de disposiciones que se adicionan a los contratos de concesión de los servicios, los convenios por obras, etc. Las regulaciones indican las condiciones de prestación, su calidad, las tarifas asociadas, y los costos de conexión y son aplicados por los entes de regulación autárquicos en términos financieros, que se relacionan con el PE mediante algunos ministerios (principalmente ligados a obras públicas), y cuyo financiamiento se efectúa mediante un porcentaje de las tarifas y asignaciones del presupuesto público.

Ciertamente la decisión en torno a los servicios regulados, bajo qué regímenes tarifarios y con qué estructura de las agencias constituye "un hecho político" (Herrero, 2002:12). Es de destacar que la literatura señala que cuando las agencias perciben la mayor parte de sus ingresos del presupuesto público anual de cada jurisdicción pueden crear "una dependencia no deseable de los entes respecto de los poderes legislativo y ejecutivo, en cuanto a la fijación y a la efectivización de las sumas presupuestadas" (Herrero, 2002: 59) y en momentos del ciclo económico recesivo y de reducción de la recaudación afectar la calidad regulatoria. Vispo (1999) destaca la importancia de evitar las distorsiones como la cooptación estándar, situación en que el regulador se desvía de su objetivo teórico de defender el "bien común", cediendo a la presión de un grupo interesado en particular y la cooptación bifronte, situación de cooptación en donde la agencia reguladora es capturada simultáneamente por la empresa que regula y por la autoridad política. 
Herrero (2002) sostiene que el financiamiento de los entes reguladores mediante una tasa de mantenimiento, control y fiscalización como parte de la tarifa pagada por los usuarios es la opción que genera mayor autarquía financiera e independencia del sector empresarial y político más allá del tipo de esquema de prestación.

El poder monopólico que detentan las empresas prestatarias en el caso de la provisión por redes (y que es una particularidad de estos servicios) -con independencia del carácter estatal de la propiedad o privado de la concesión- adquiere un estado crítico por la esencialidad de los servicios y las externalidades asociadas. La presencia de monopolios naturales y locales en la prestación, con una demanda fuertemente inelástica en relación con el precio, realza la importancia de la regulación económica activa del Estado orientada a fijar precios máximos, niveles cobertura y calidades adecuadas para la provisión, lo cual indirectamente incide sobre los niveles de inversión necesarios. Así como de regulación antimonopólica de los actos de concentración y de las prácticas anticompetitivas que pudieran implementar las empresas con poder de mercado sean nuevamente, de propiedad estatal o privada (Forcinito, 2005).

\subsection{Los esquemas tarifarios vigentes}

En Argentina, como en la región en general, los recursos financieros que perciben las empresas, más allá de la forma jurídica que adopten, contemplan fuentes propias y externas y asumen un grado de criticidad importante por las características estructurales de los servicios (que requieren una proporción de costos fijos elevados respecto de los totales y demanda de fuertes gastos de mantenimiento). Esto implica que requieren inversiones en capital fijo que no pueden reasignarse luego a otros sectores y que tienen una lenta recuperación de las inversiones.

Puntualmente en lo que concierne a las inversiones vinculadas al mantenimiento de las ins- talaciones y expansión de las infraestructuras cobra importancia el régimen tarifario vigente de los servicios.

La regulación de las tarifas puede ser utilizada y lo ha sido en el caso de Argentina como instrumento de control de otras variables de la economía (por ejemplo la inflación y el consumo). Los recursos endógenos con los que cuentan las empresas se vinculan directamente con las tarifas. Los esquemas de fijación de los precios a través de la determinación directa o indirecta de la tasa de ganancia apuntan a mantener la sustentabilidad de la prestación como la inversión en capital fijo que posibilite el mantenimiento y expansión de las infraestructuras tendientes a incorporar a la población desabastecida (Azpiazu y Forcinito, 2004:36).

Ciertamente, el régimen tarifario "depende de factores sociales, económicos, de fuentes, etc. pero en definitiva, lo que se busca es que la facturación resultante produzca los ingresos suficientes para solventar los gastos de operación, mantenimiento, amortizaciones, depreciaciones y beneficios de la empresa, asociado a una tarifa acorde con el poder adquisitivo de la población" (Savid, 1997: 36).

Por lo que, la política tarifaria del sector tiende a distribuir los costos (fijos y variables) de la prestación, mantenimiento y expansión de los servicios entre los distintos usuarios y al nivel medio de la tarifa que refiere al nivel de recuperación de los costos (Núñez, 2011).

En general, los niveles tarifarios se encuentran limitados jurídicamente por el principio de razonabilidad tarifaria que involucra una tasa de ganancia equivalente a la que rige para otras actividades de riesgo similar, cualquiera sea el sistema que se utilice (Solanes, 1999).

Los esquemas tarifarios presentes en el sector refieren a precio tope, tasa de retorno o combinaciones híbridas. Las características de las modalidades regulatorias son: 
$\checkmark \quad$ Esquema Price cap (precio tope) que prevé la fijación de un precio base máximo (acordado por el poder concedente) el cual otorga a la empresa una ganancia razonable; el mismo se ajusta en forma periódica con la aplicación de un índice de precios domésticos (que tiende a reflejar las variaciones de los costos) al cual se le sustrae un factor de eficiencia con el propósito de transferir a los usuarios y consumidores los incrementos de productividad de la empresa y luego de un periodo se realizan revisiones tarifarias donde esas ganancias se trasladan a los usuarios.

$\checkmark$ Tasa de retorno (costo del servicio) que refiere a la determinación por parte del poder concedente de la rentabilidad sobre el capital invertido para el prestador. Lo que genera que se trasladen a las tarifas los incrementos de costos relevantes y mantiene constante la rentabilidad del prestador (Saravia, 2010). Este mecanismo, al mantener establece la tasa de rentabilidad, tiene como ventaja central la corrección del sesgo a la desinversión que caracteriza a las empresas del sector en tanto proporciona estabilidad a la tasa de rentabilidad sobre el capital empleado de largo plazo (Vickers y Yarrow, 1991).

$\checkmark \quad$ Mixto o híbridos que representa a un tipo de mecanismo que recupera o combina el price cap con incrementos por la variación de costos.

Al interior del país, considerando la información disponible respecto a la prestación de los servicios de agua y saneamiento al AMBA (a cargo del Estado nacional) y las provincias de Catamarca, Tucumán, PBA, Salta, Jujuy, Santiago del Estero, Corrientes, Misiones, Córdoba, Río Negro y La Rioja se observa que aunque el sistema tarifario más generalizado es el Price cap, en la práctica la regulación tarifaria contiene un esquema mixto, en tanto estas jurisdicciones señaladas autorizan revisiones periódicas que consideran la variación de los costos (como se observa en las Tablas 1, 2 y 3).

Tabla 1

Agencias regulatorias de los servicios en Argentina (2015)

\begin{tabular}{|c|c|c|c|c|}
\hline Provincia & $\begin{array}{c}\text { Organismo de } \\
\text { Regulación y/o Control }\end{array}$ & Características & $\begin{array}{l}\text { Marco } \\
\text { Regulatorio }\end{array}$ & Sistema tarifario \\
\hline AMBA & $\begin{array}{l}\text { Ente Regulador del Agua } \\
\text { y Saneamiento (ERAS) }\end{array}$ & $\begin{array}{l}\text { Ente autárquico e interjuris- } \\
\text { diccional }\end{array}$ & Ley 26.221 & $\begin{array}{l}\text { Se mantiene con las tarifas con- } \\
\text { geladas desde } 2002 \text {. El Estado } \\
\text { nacional financia el déficit. }\end{array}$ \\
\hline $\begin{array}{l}\text { Buenos } \\
\text { Aires }\end{array}$ & $\begin{array}{l}\text { Organismo de Control } \\
\text { del Agua de Buenos } \\
\text { Aires }\end{array}$ & $\begin{array}{l}\text { Ente autárquico en la órbita } \\
\text { del Ministerio de Infraestruc- } \\
\text { tura. }\end{array}$ & $\begin{array}{l}\text { Decreto } \\
873 / 2003\end{array}$ & $\begin{array}{l}\text { Sistema hibrido establecido } \\
\text { por decreto del PEP }\end{array}$ \\
\hline Catamarca & $\begin{array}{l}\text { Ente Regulador de los } \\
\text { Servicios Públicos y otras } \\
\text { concesiones }\end{array}$ & $\begin{array}{l}\text { Ente Autárquico y en la órbi- } \\
\text { ta del Ministerio de Hacien- } \\
\text { da y Finanzas }\end{array}$ & Ley 4836 & $\begin{array}{l}\text { Price cap con revisiones de } \\
\text { costos }\end{array}$ \\
\hline Córdoba & $\begin{array}{l}\text { Ente Regulador de los } \\
\text { Servicios Públicos }\end{array}$ & $\begin{array}{l}\text { Ente Autárquico que se re- } \\
\text { laciona con PE a través del } \\
\text { Ministerio de Obras Públicas }\end{array}$ & Ley $N^{\circ} 8835$ & $\begin{array}{l}\text { Price cap con un precio máxi- } \\
\text { mo por tres años y luego revi- } \\
\text { sión periódica. }\end{array}$ \\
\hline Corrientes & $\begin{array}{l}\text { Administración de Obras } \\
\text { Sanitarias Corrientes }\end{array}$ & $\begin{array}{l}\text { Ente autárquico de derecho } \\
\text { público y privado se relacio- } \\
\text { na con PE a través del Mi- } \\
\text { nisterio de Obras y Servicios } \\
\text { Públicos }\end{array}$ & $\begin{array}{l}\text { Leyes } 3573 \\
\text { y } 3979\end{array}$ & $\begin{array}{l}\text { Price cap con revisiones de } \\
\text { costos operativos. }\end{array}$ \\
\hline
\end{tabular}

Fuente: Elaboración propia en base a la información de los entes de regulación y Sarabia (2010) 


\section{Tabla 2}

Agencias regulatorias de los servicios en Argentina (2015)

\begin{tabular}{|c|c|c|c|c|}
\hline Provincia & $\begin{array}{c}\text { Organismo de Regulación } \\
\text { y/o Control }\end{array}$ & Características & $\begin{array}{c}\text { Marco } \\
\text { Regulatorio }\end{array}$ & Sistema tarifario \\
\hline Formosa & $\begin{array}{l}\text { Ente regulador de obras y } \\
\text { servicios públicos }\end{array}$ & $\begin{array}{l}\text { Ente autárquico de derecho } \\
\text { público con capacidad para } \\
\text { actuar privada y pública- } \\
\text { mente }\end{array}$ & Ley $N^{\circ} 1.142$ & No se tiene información \\
\hline Jujuy & $\begin{array}{l}\text { Dirección de Servicios Pú- } \\
\text { blicos y otras concesiones }\end{array}$ & $\begin{array}{l}\text { Ente Autárquico que se re- } \\
\text { laciona con PE mediante el } \\
\text { Ministerio de Infraestructura } \\
\text { y Planificación }\end{array}$ & Ley N 4937 & $\begin{array}{l}\text { Price cap con revisiones } \\
\text { de costos }\end{array}$ \\
\hline La Rioja & $\begin{array}{l}\text { Ente Único de Control de } \\
\text { las privatizaciones de la } \\
\text { Provincia de La Rioja }\end{array}$ & $\begin{array}{l}\text { Ente Autárquico que con- } \\
\text { trala el servicio de agua y } \\
\text { energía }\end{array}$ & Ley 6.281 & Price cap \\
\hline Mendoza & $\begin{array}{l}\text { Ente Provincial del Agua y } \\
\text { de Saneamiento }\end{array}$ & $\begin{array}{l}\text { Ente autárquico de derecho } \\
\text { público y privado }\end{array}$ & $\begin{array}{l}\text { Decreto } \quad N^{\circ} \\
2648 / 10\end{array}$ & $\begin{array}{l}\text { Price cap por } 5 \text { años y se } \\
\text { contempla la posibilidad } \\
\text { de revisar la tarifa durante } \\
\text { este lapso }\end{array}$ \\
\hline Misiones & $\begin{array}{l}\text { Ente Provincial Regulador } \\
\text { de Agua y Cloacas }\end{array}$ & $\begin{array}{l}\text { Ente Autárquico y su rela- } \\
\text { ción se efectúa con el PE } \\
\text { mediante el Ministerio } \\
\text { de Obras Públicas }\end{array}$ & Ley 3391 & $\begin{array}{l}\text { Sistema de revisiones de } \\
\text { costos }\end{array}$ \\
\hline
\end{tabular}

Fuente: Elaboración propia en base a la información de los entes de regulación y Sarabia (2010)

\section{Tabla 3}

\section{Agencias regulatorias de los servicios en Argentina (2015)}

\begin{tabular}{|c|c|c|c|c|}
\hline Provincia & $\begin{array}{c}\text { Organismo de Regulación } \\
\text { y/o Control }\end{array}$ & Características & $\begin{array}{l}\text { Marco } \\
\text { Regulatorio }\end{array}$ & Sistema tarifario \\
\hline Rio Negro & $\begin{array}{l}\text { Departamento Provincial del } \\
\text { Agua }\end{array}$ & $\begin{array}{l}\text { Ente autárquico dependiente } \\
\text { del PE }\end{array}$ & Ley 3188 & No se tiene información \\
\hline Salta & $\begin{array}{l}\text { Ente Regulador de los } \\
\text { Servicios Públicos }\end{array}$ & $\begin{array}{l}\text { Ente autárquico de derecho } \\
\text { público y privado vinculado } \\
\text { con el PE mediante el Minis- } \\
\text { terio de Desarrollo Económi- } \\
\text { co }\end{array}$ & Ley 6835 & $\begin{array}{l}\text { Tarifas máximas con re- } \\
\text { conocimiento mensual } \\
\text { por variaciones en los } \\
\text { costos. }\end{array}$ \\
\hline Santa Fe & $\begin{array}{l}\text { Ente regulador de servicios } \\
\text { sanitarios }\end{array}$ & $\begin{array}{l}\text { Ente autárquico con capaci- } \\
\text { dad de derecho público. }\end{array}$ & Ley 11.220 & $\begin{array}{l}\text { Price cap con revisiones } \\
\text { de costos }\end{array}$ \\
\hline $\begin{array}{l}\text { Santiago del } \\
\text { Estero }\end{array}$ & $\begin{array}{l}\text { Ente Regulador de los Servi- } \\
\text { cios de Santiago del Estero }\end{array}$ & $\begin{array}{l}\text { Ente autárquico descentrali- } \\
\text { zado }\end{array}$ & Ley 6.225 & $\begin{array}{l}\text { Sistema hibrido con un } \\
\text { precio máximo y se habi- } \\
\text { litan revisiones tarifarias }\end{array}$ \\
\hline Tucumán & $\begin{array}{l}\text { Ente Regulador del Servicio } \\
\text { de Agua y Cloacas de Tucu- } \\
\text { mán }\end{array}$ & $\begin{array}{l}\text { Ente Autárquico dependien- } \\
\text { te del P.E. relacionándose } \\
\text { con el Ministerio de Desa- } \\
\text { rrollo Productivo }\end{array}$ & $\begin{array}{l}\text { Leyes } 6445 \text { y } \\
6.529\end{array}$ & $\begin{array}{l}\text { Price cap con revisiones } \\
\text { de costos. }\end{array}$ \\
\hline
\end{tabular}

Fuente: Elaboración propia en base a la información de los entes de regulación y Sarabia (2010) 
La mayoría de los casos, anteriormente, señalados contienen también un esquema de subsidios cruzados entre tipo de usuarios residenciales y no residenciales, entre zonas, antigüedad y calidad de los inmuebles y un programa de tarifa social que refiere a un subsidio focalizado a hogares en situación de vulnerabilidad (pobreza, discapacidad, etc.).

Más allá de lo cual los componentes de las tarifas no consideran un conjunto de costos asociados a la propia conservación de las fuentes de abastecimiento de agua sea superficial o subterránea y el saneamiento de las cuencas receptoras de efluentes domésticos.
Cabe destacar que la gestión por cuencas en Argentina, como explica Pochat (2005) parece ser aceptada como un "concepto estrictamente técnico" y su "receptividad en las institucionalidades nacionales, su vinculación a la política hídrica y la determinación de competencias y recursos, todavía dejan muchos aspectos en suspenso". 7

Puntualmente para 2010 los niveles de cobrabilidad de los servicios en promedio en estas jurisdicciones alcanzaban al 88,3\% siendo superior en aquellas jurisdicciones con prestaciones privadas Misiones, Corrientes y Córdoba como se observa en el Gráfico 1.

\section{Gráfico 1}

Cobrabilidad de los servicios de agua y saneamiento, 2010

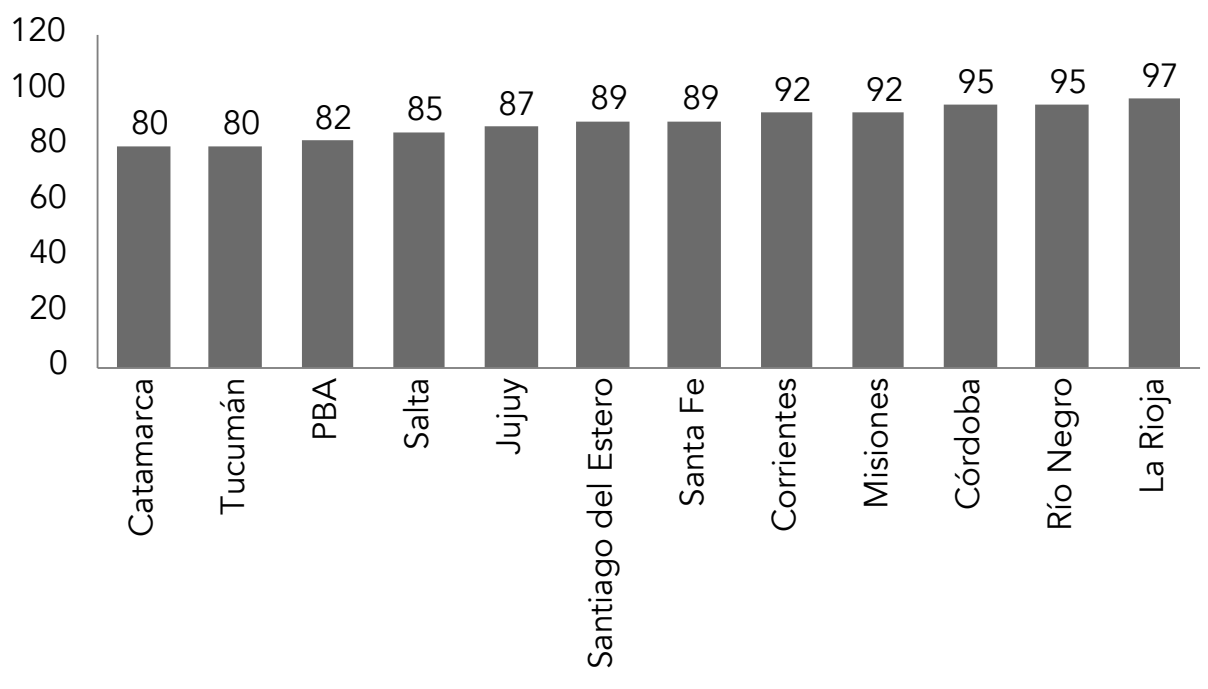

Cobrabilidad

Fuente: Elaboración propia sobre la información de Sarabia (2010)

\subsection{La cobertura de los servicios}

Desde 1990, el acceso al agua por red pública presenta mejoras importantes en Argentina, sin embargo, aún no ha logrado alcanzar la meta propuesta por el Estado nacional en el marco de los Objetivos del Milenio de reducir a dos terceras partes la proporción de población del país sin acceso al agua (alcanzar al 90\%). Se observan mayores dificultades en la meta de reducir a dos terceras partes la proporción de población sin acceso a las cloacas (alcanzar el 75\%) que se encontraba por debajo de la cobertura de agua. Esta dificultad está también presente en la región en la que los niveles de acceso al agua potable por redes son mayores a los de acceso a cloacas.

7 En Argentina los avances en lo que concierne a la gestión por cuencas están enfocados en la conformación de entidades interjurisdiccionales que suponen la planificación 
Los últimos datos de cobertura de los servicios para todo el país refieren a la medición de 2010 del Censo Nacional de Población, Hogares y Viviendas que mostraron mejoras importantes respecto a 2001. El Censo dio cuenta que la cobertura de agua alcanzaba al $84 \%$ de los hogares y al $53,2 \%$ en las cloacas y era he- terogénea en las jurisdicciones. Las jurisdicciones con mayores niveles de hogares sin acceso a ambos servicios centralizados afectaban a las provincias de Chaco, Santiago del Estero, PBA, Formosa y a las provincias de Misiones y en Córdoba y San Juan en lo que refiere a cloacas, como se observa en los Gráficos 2 y 3.

\section{Gráfico 2}

Cobertura de hogares con acceso al agua 2001-2010

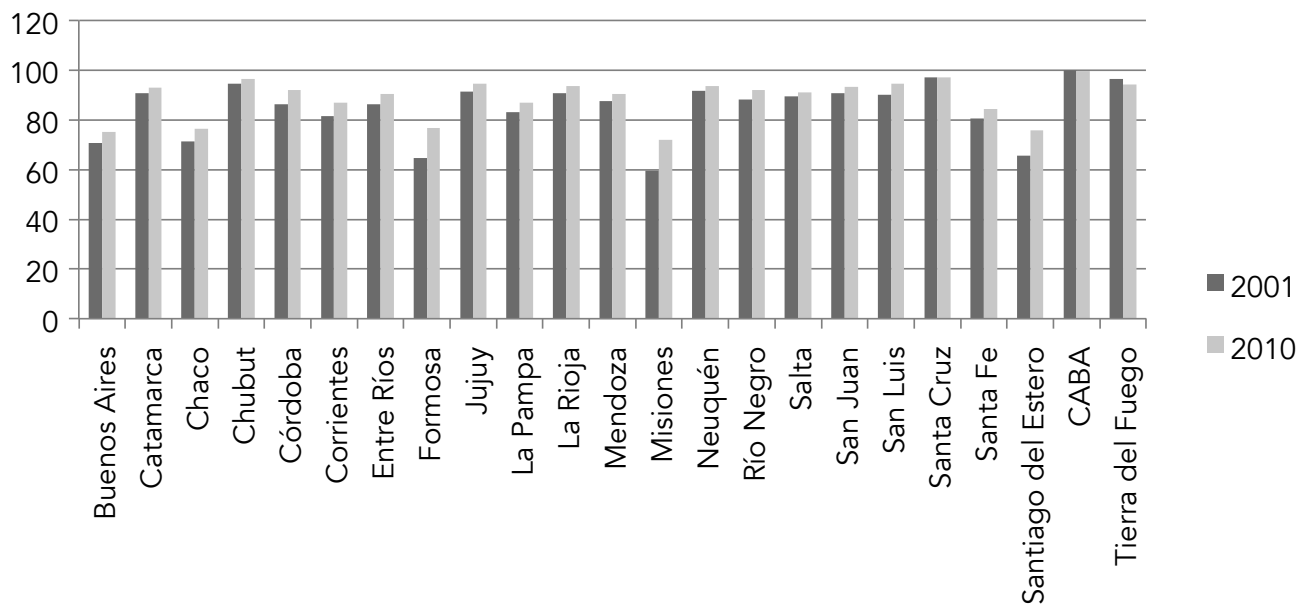

Fuente: Elaboración propia en base a los datos del INDEC (2010)

\section{Gráfico 3}

Cobertura de hogares con acceso al agua 2001-2010

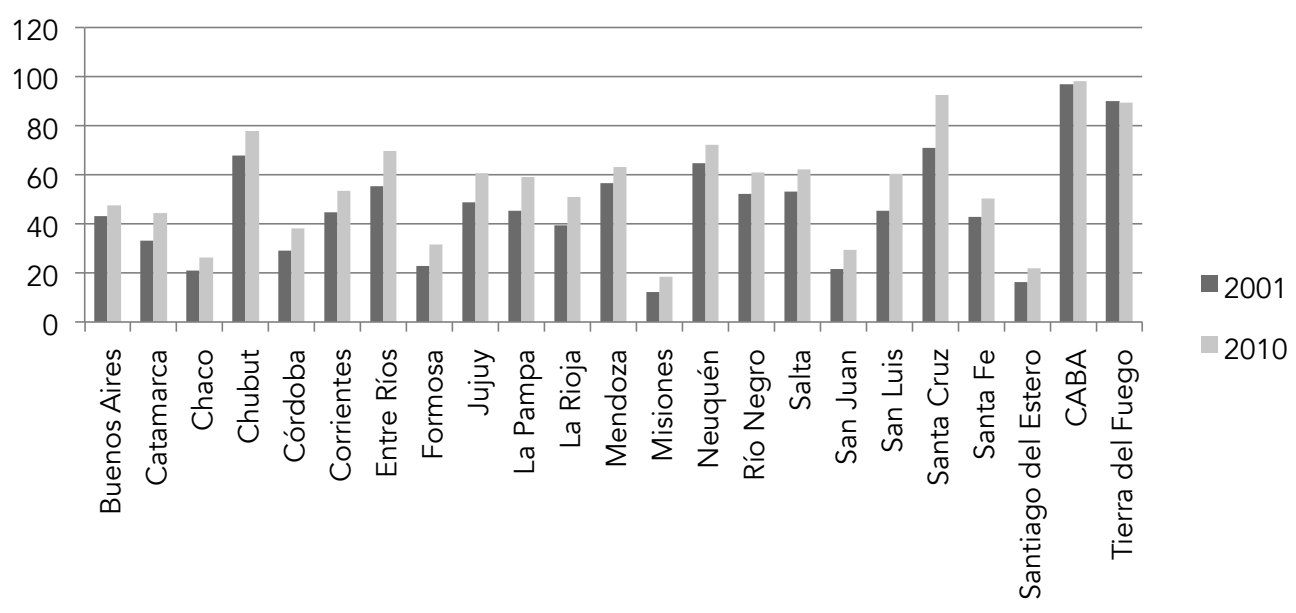

Fuente: Elaboración propia en base a los datos del INDEC (2010)

conjunta entre jurisdicciones sobre los recursos compartidos; entidades provinciales y a nivel internacional en el caso de las fuentes compartidas con otros países. 
Es de destacar que el sector de agua y saneamiento es el que demanda inversiones que son muy importantes por los costos hundidos que tiene que enfrentar para la ampliación de la capacidad productiva de las plantas de tratamiento de agua y de los efluentes, el tendido de ductos, etc.

Las jurisdicciones del país que tienen menores niveles de cobertura organizan los servicios mediante modelos de gestión disímiles, en el caso de la PBA y parte del AMBA las empresas fueron re-estatizadas y están a cargo de sociedades anónimas con mayoría accionaria del Estado. En el caso de Chaco, más allá de algunos intentos de privatización que experimentó en los años noventa, los servicios siempre se han mantenido en la esfera del Estado provincial en la mayor parte del territorio. En el caso de las provincias de Córdoba, Misiones y Santiago del Estero los servicios aún están gestionados mediante empresas privadas. El Gráfico 4 permite observar el comportamiento del gasto público destinado al sector en las jurisdicciones más afectadas por los problemas de cobertura.

\section{Gráfico 4}

Gasto público en agua y saneamiento durante 1993-2009

(precios constantes, IPI Base 1993=100)

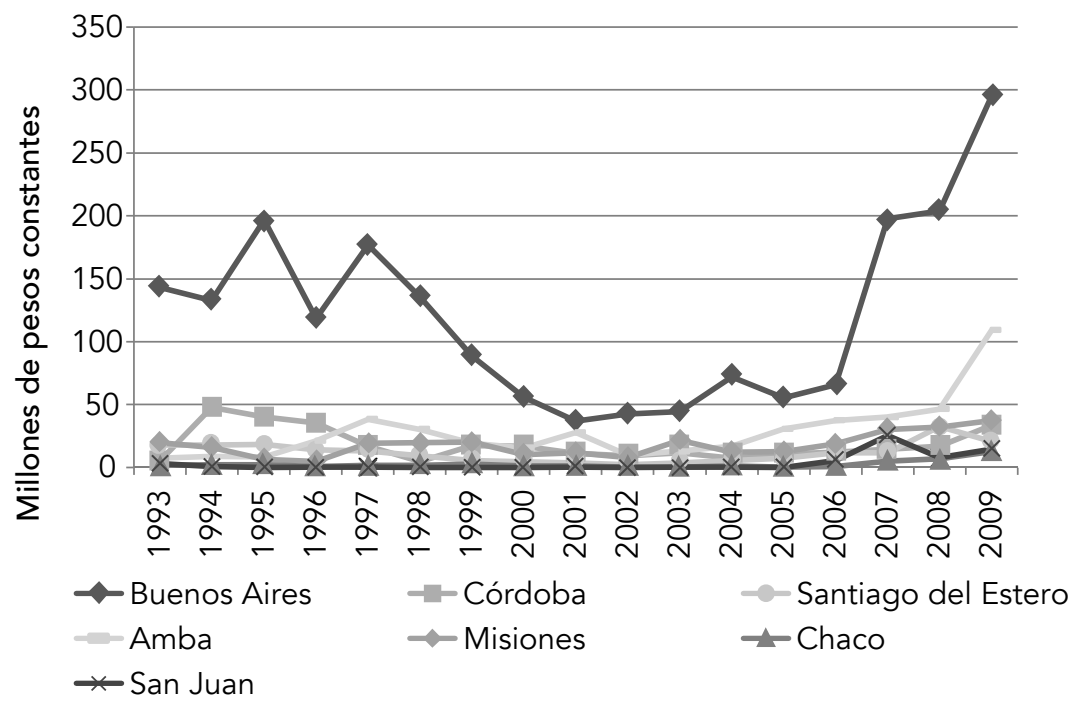

Fuente: Elaboración propia en base a la información de la Dirección de Análisis de Gasto Público y Programas Sociales - Secretaría de Política Económica (2010) 


\section{Conclusiones}

En Argentina los servicios de agua y saneamiento han atravesado cambios profundos desde 1980 con el proceso de descentralización y transferencia a las jurisdicciones provinciales y de procesos de privatización (que fueron de la mano de la concepción del agua como una simple mercancía, es decir resaltando sólo su valor económico). Desde 2002 el país experimentó un proceso de reestatizaciones de los servicios en las principales jurisdicciones que incluyen la PBA, el AMBA y Santa $\mathrm{Fe}$, pero coexisten múltiples formas jurídicas de prestación que incluyen sociedades anónimas privadas y cooperativas.

El sector mantiene así agencias regulatorias que fueron constituidas bajo la lógica de regular a capitales privados y que en las jurisdicciones que los servicios fueron re-estatizados no se han modificado estructuralmente. Al igual que los marcos regulatorios que continúan avalando en algunas provincias el corte de los servicios frente a situaciones de mora en los pagos.

\section{Desafíos}

El sector de agua y saneamiento tiene entre sus desafíos centrales:

Avanzar hacia el logro de los Objetivos del Milenio y más aún continuar sosteniendo y ampliando las inversiones para aproximarse a la universalización del acceso a los servicios.

Mejorar la calidad de las prestaciones de los servicios en términos de los patrones de la calidad bacteriológica y química del agua y el tratamiento de los efluentes, lo cual demanda de marcos regulatorios que consideren la sustentabilidad financiera, técnica y ambiental y un conjunto de políticas que se mantengan más allá del cambio de los gobiernos. Esto adquiere relevancia en la coyuntura actual del país donde se presenta un recambio de gobierno.
Avanzar en lineamientos claros y debatidos entre actores estatales y no estatales que consideren la política de agua y saneamiento dentro de la política hídrica en general.

Discutir la estructura tarifaria de los servicios de agua y saneamiento e internalizar los costos asociados a la gestión hídrica de las cuencas.

Promover la participación de organismos no estatales en la política sectorial más allá de su representación en vocalías de los entes de regulación.

Fortalecer el control del vuelco de los efluentes industriales en los cursos de agua.

Promover y conformar instancias de coordinación de las acciones en el sector y de cooperación entre las empresas prestatarias entre jurisdicciones.

\section{Referencias}

Abdala, M y Spiller, P. 1999. Instituciones, contratos y regulación en Argentina. Editorial Temas, Buenos Aires.

Abihaggle, C. y Day J. 2004. Agua y sociedad. Un ensayo económico sobre la política hídrica. EDIUNC, Mendoza.

Academias Nacionales de Ingeniería, Ciencias Económicas y Ciencias exactas, Físicas y Naturales 2011. La cuestión del agua. Algunas consideraciones sobre el estado de situación de los recursos hídricos de la Argentina, Buenos Aires.

Azpiazu, D. y Forcinito, K. 2004. "Historia de un fracaso: la privatización del sistema de agua y saneamiento en el Área Metropolitana de Buenos Aires" en Azpiazu, D., Catenazzi, A. y Forcinito, K. (2004). Recursos públicos, negocios privados: agua potable y saneamiento en el Área Metropolitana de Buenos Aires. Serie Informes de Investigación Nº 19. Universidad Nacional de General Sarmiento, Los Polvorines. 
Bella, A. y Hure, M. 2007. El agua. Conocerla para cuidarla. UNR-Editora, Rosario.

Bruzzone, E. 2009. Las guerras del agua: América del sur, en la mira de las grandes potencias. Capital intelectual, Buenos Aires.

Busch, T. 2013. Desarrollo y ecopolítica. Los grandes debates de la tecnología, el ambiente y la sociedad. Lenguaje Claro Editora, Carapachay.

Constitución de la Nación Argentina. Consultado el 25 agosto 2015. Disponible en http://infoleg.mecon.gov.ar/infoleglnternet/anexos/0-4999/804/norma.htm

Corrales, M. 2004. "Gobernabilidad de los servicios de agua potable y saneamiento en América Latina" en Revista REGA.

Dirección de Análisis de Gasto Público y Programas Sociales. 2010. Secretaría de Política Económica. Gasto de los gobiernos provinciales y GCBA. Ministerio de Economía de la Nación, Buenos Aires.

Forcinito, K. (2005): Los sectores de infraestructura para la prestación de servicios públicos en la Argentina. Lineamientos generales de regulación pública, Buenos Aires, inédito.

Global Water Partnership-GWP. 2008. Principios de Gestión Integrada de los Recursos Hidricos. Bases para el desarrollo de planes nacionales. (Documento GWP). Consultado el 12 agosto 2015. Disponible en

http://www.gwp.org/Global/GWPCAm Files/Bases\%20para\%20el\%20Desarrollo\%20de\%20Planes\%20Nacionales.pdf

Hantke-Domas, M. y Jouravlev, A. 2011. Lineamientos de política pública para el sector de agua y saneamiento. CEPAL, Santiago de Chile.
Herrero, F. 2002. La regulación provincial de los servicios públicos en Argentina. Ediciones de la Universidad Nacional de Lanús, Lanús.

Instituto Nacional de Estadísticas y Censos. 2001. Censo Nacional de Población, Hogares y Viviendas de 2001. Consultado el 11 agosto 2015. Disponible en http://www.indec.gov.ar/micro sitios/ webcenso/censo2001s2/ampliada index. asp?mode $=01$

Instituto Nacional de Estadísticas y Censos .2010. Censo Nacional de Población, Hogares y Viviendas de 2010. Consultado el 11 agosto 2015. Disponible en http:// www.censo2010.indec.gov.ar/archivos/ censo2010 tomo1.pdf

Ley Nacional Consejo Hídrico Federal $\mathrm{N}^{\circ}$ 26.438. Consultado el 4 septiembre 2015. Disponible en http://www. infoleg.gov.ar/infoleglnternet/anexos/145000-149999/149387/norma.htm

Ley Nacional de Régimen de Gestión Ambiental de Aguas $N^{\circ}$ 25.688. Consultado el 4 septiembre 2015. Disponible en http://infoleg.mecon.gov.ar/infoleglnternet/anexos/80000-84999/81032/norma.htm

Ley Nacional de Sociedades cooperativas $\mathrm{N}^{\circ}$ 11.388. Consultado el 4 septiembre 2015. Disponible en http://www.infoleg.gob.ar/ infoleglnternet/verNorma.do?id=96249

López, A. y Felder, R. 2006. "La regulación estatal ¿servicio público o fallas del mercado? Algunas reflexiones sobre los criterios de regulación" en Realidad Económica N 163, Buenos Aires

Mutuberría Lazarini, V. 2007. Los servicios públicos urbanos como medios colectivos para la producción y reproducción de la vida de los sujetos en sociedad desde la perspectiva de la economía social: análisis de experiencias de gestión colectiva en el Gran Buenos Aires. Buenos Aires. Tesis de maes- 
tría en economía social, Universidad Nacional de General Sarmiento, Los Polvorines

Núñez, A. 2006. Lo que el agua (no) se llevó... Política urbana, estado del poder, violencia e identidades sociales. Mar del Plata, entre siglos. Tesis Doctoral en Ciencias Sociales FLACSO.

Ordoque Urcelay, M. 2007. Servicios de agua potable y alcantarillado en la ciudad de Buenos Aires, Argentina: factores determinantes de la sustentabilidad y el desempeño. Serie Recursos Naturales e Infraestructura, $N^{\circ} 126, C E P A L$, Santiago de Chile.

Picolotti, J. 2003. "The Right to Water in Argentina". Informe del CEDHA para Rights and Humanity. Consultado el $10 \mathrm{sep}-$ tiembre 2015. Disponible en http://www. righttowater.info/wp-content/uploads/ argentina CS.pdf

Pirez, P. 2001. Gestión de servicios urbanos y exclusión en América Latina. Unión Iberoamericana de Municipalistas Granada. Síntesis N 10, Granada.

Rojas Oruste, F. 2010. Gobernabilidad y gobernanza. De la teoría a la práctica. Aplicación a los servicios de agua y saneamiento. ANEAS, DF México.

Saravia, J. 2010. Conclusiones de los Seminarios de Tarifas y Sistemas Tarifarios de AFERAS. AFERAS, Buenos Aires
Savid, R. 1997. "La gestión comercial de los procesos de Transformación de las empresas de saneamiento" en COFES. Modelos de gestión comercial en empresas de saneamiento. Buenos Aires.

Schifini, J. 1998. Panorama general de las transformaciones en Argentina. Documento Técnico $N^{\circ} 5$, Consejo Federal de Entidades de Servicios Sanitarios, Buenos Aires.

Solanes, M. 1999. Servicios públicos y regulación. Consecuencias legales de las fallas de mercado. Serie Recursos Naturales e Infraestructura $N^{\circ}$ 2, CEPAL, Santiago de Chile.

Solanes, M. y Jouravlev, A. (2005): Integrando economía, legislación y administración en la gestión del agua y sus servicios en América Latina y el Caribe, CEPAL, Santiago de Chile.

Vickers, J. y Yarrow, J. 1991. Un análisis económico de la privatización. Fondo de Cultura Económica, Gran Bretaña.

Vispo, A. 1999. Los entes de regulación. Problemas de diseño y contexto. Aportes para un urgente debate en la Argentina, Editorial Norma, Buenos Aires. 